\title{
Flavonoids activate pregnane $\times$ receptor-mediated CYP3A4 gene expression by inhibiting cyclin-dependent kinases in HepG2 liver carcinoma cells
}

\author{
Hanqing Dong ${ }^{+1,2}$, Wenwei Lin+1, Jing Wu ${ }^{1}$ and Taosheng Chen*1
}

\begin{abstract}
Background: The expression of the drug-metabolizing enzyme cytochrome P450 3A4 (CYP3A4) is regulated by the pregnane $\times$ receptor $(P X R)$, which is modulated by numerous signaling pathways, including the cyclin-dependent kinase (Cdk) pathway. Flavonoids, commonly consumed by humans as dietary constituents, have been shown to modulate various signaling pathways (e.g., inhibiting Cdks). Flavonoids have also been shown to induce CYPs expression, but the underlying mechanism of action is unknown. Here, we report the mechanism responsible for flavonoid-mediated PXR activation and CYP expression.

Results: In a cell-based screen designed to identify compounds that activate PXR-mediated CYP3A4 gene expression in HepG2 human carcinoma cells, we identified several flavonoids, such as luteolin and apigenin, as PXR activators. The flavonoids did not directly bind to PXR, suggesting that an alternative mechanism may be responsible for flavonoidmediated PXR activation. Consistent with the Cdk5-inhibitory effect of flavonoids, Cdk5 and p35 (a non-cyclin regulatory subunit required to activate $(\mathrm{dk} 5)$ were expressed in HepG2. The activation of C dk5 attenuated PXRmediated CYP3A4 expression whereas its downregulation enhanced it. The Cdk5-mediated downregulation of CYP3A4 promoter activity was restored by flavonoids, suggesting that flavonoids activate PXR by inactivating Cdk5. In vitro kinase assays showed that C $\mathrm{dk} 5$ directly phosphorylates PXR. The Cdk kinase profiling assay showed that apigenin inhibits multiple Cdks, suggesting that several Cdks may be involved in activation of PXR by flavonoids.

Conclusions: Our results for the first time link the stimulatory effect of flavonoids on CYP expression to their inhibitory effect on Cdks, through a PXR-mediated mechanism. These results may have important implications on the pharmacokinetics of drugs co-administered with herbal remedy and herbal-drug interactions.
\end{abstract}

\section{Background}

The pregnane $\times$ receptor (PXR) is a key xenobiotic receptor that regulates the metabolism and excretion of xenobiotics and endobiotics by regulating the expression of drug-metabolizing enzymes and drug transporters $[1,2]$. Expression of PXR target gene is regulated by binding of PXR to its promoter region, such as that of cytochrome P450 3A4 (CYP3A4), a key enzyme that catalyzes the

* Correspondence: taosheng.chen@stjude.org

1 Department of Chemical Biology and Therapeutics, St. Jude Children's Research Hospital, 262 Danny Thomas Place, Memphis, TN 38105, USA

+ Contributed equally

Full list of author information is available at the end of the article metabolism of more than $50 \%$ of all clinically prescribed drugs [3]. Changes in the expression of CYP3A4 affect drug metabolism and alter the therapeutic and toxicologic responses to drugs, which may in turn lead to adverse drug interactions.

The activity of PXR is regulated not only by direct ligand binding [4] but also by various cell signaling pathways [5], such as those mediated by protein kinase $C$ (PKC) [6], protein kinase A (PKA) [7,8], cyclin-dependent kinase 2 (Cdk2) [9], 70kDa form of ribosomal protein S6 kinase (p70 S6K) [10], forkhead in rhabdomyosarcoma (FKHR) [11], and nuclear factor $\mathrm{kB}$ (NF-kB) [12-14]. 
Flavonoids - secondary metabolites found ubiquitously in plants - are the most common group of polyphenolic compounds consumed by humans as dietary constituents [15]. Thousands of naturally occurring flavonoids, such as flavones and isoflavones, have been characterized [16]. Flavonoids have been reported to have anti-allergic, antiinflammatory, anti-microbial and anti-cancer activities $[17,18]$. The widespread use of flavonoids, coupled with their potentially beneficial effects, has triggered studies on the mechanism by which they modulate signaling pathways.

Natural flavonoids have been shown to inhibit Cdk1, Cdk2 [19], and Cdk5 [20]. Most Cdks, including Cdk1 and Cdk2, are involved in cell cycle regulation and require the binding of cyclins for their activation. However, the activation of Cdk5 requires one of the two noncyclin regulatory subunits p35 or p39, which have $57 \%$ amino acid homology. p35 can be converted in a $\mathrm{Ca}^{2+}$ dependent manner to $\mathrm{p} 25$, a highly active and stable proteolytic product $[21,22]$. The protease calpain catalyzes the cleavage of p35, and this reaction can be effectively inhibited by specific inhibitors of calpain such as calpeptin $[23,24]$. Cdk5 is not involved in cell cycle progression, and is expressed in all tissues, but its levels of expression and activity are highest in the nervous system [21,25]. The expressions of p35 and p39 are also highest in the nervous system. Although Cdk5 has been mainly implicated in early development of the central nervous system (CNS) and maintenance of neuronal architecture $[21,26]$, the expression and regulatory activity of Cdk5/p35 have also been reported in several non-CNS tissues such as lens epithelia [27], muscle tissues [28], hepatoma cells [25], adipose tissues [29], and male reproductive system [30].

The widespread use of flavonoids has triggered studies to investigate their effects on drug metabolism and herbal-drug interactions. Recently, flavonoids have been shown to induce CYP expression through PXR [31-36], but the mechanism of flavonoids-mediated PXR activation and CYP induction remain unknown.

Because the function of PXR can be modulated by cellular signaling pathways, we used a cell-based screening approach in this study to identify compounds with known bioactivities that activate PXR-mediated gene expression. By screening a library of known bioactive compounds, we identified a series of flavonoids that are PXR activators. Since these flavonoids did not directly bind to PXR, and flavonoids might inhibit Cdk5, we studied the effect of flavonoids on the activity of Cdk5/p35 and the regulation of PXR by Cdk5 in order to determine the possible role of flavonoids in regulating PXR-mediated gene expression of CYP3A4.

\section{Results}

Flavonoids activate PXR-mediated CYP3A4 gene expression

By screening a library of 3200 compounds with known bioactivity in the human carcinoma cell line HepG2 stably transfected with PXR and CYP3A4-luc, which was previously used to detect the activation PXR [9], we identified a series of flavonoids as potent activators of PXRmediated CYP3A4 promoter activation (Fig. 1). These flavonoids included flavones luteolin, apigenin (Fig. 1A), and chrysin (Fig. 1B) and isoflavones daidzein, biochanin A, prunetin, and genistein (Fig. 1B). Rifampicin, a human PXR agonist, was used as a control in this assay, and had an $\mathrm{EC}_{50}$ of $1.3 \mu \mathrm{M}$. Compared with the activation of PXR by rifampicin at $2 \mu \mathrm{M}$ (a concentration at which PXR is activated without causing cytotoxicity), some flavonoids were more potent at activating PXR at high concentrations. For example, luteolin at $40 \mu \mathrm{M}$ was 7 times more effective than $2 \mu \mathrm{M}$ rifampicin in activating PXR. Under the same assay conditions and compound treatment time $(24 \mathrm{~h})$ as the PXR transactivation assay described above, no significant cytotoxicity was detected for all flavonoids tested (data not shown).

To determine whether the flavonoids activate PXR by directly binding to it, we tested 3 flavonoids (chrysin, luteolin, and apigenin) in a PXR-binding assay. Although the potent PXR agonist SR-12813 bound strongly (Fig. $2 \mathrm{~A} ; \mathrm{IC}_{50}=42 \mathrm{nM}$ ) to PXR, chrysin did not bind to PXR at all concentrations tested (Fig. 2A). Luteolin and apigenin did not bind to PXR at or below $10 \mu \mathrm{M}$. However, below $10 \mu \mathrm{M}$, they strongly activated PXR (more than $50 \%$ of the activity from $2 \mu \mathrm{M}$ of rifampicin) (Fig. 1A). These data suggest that mechanisms other than direct PXR binding might be responsible for chrysin-, luteolin-and apigenin-mediated PXR activation.

\section{Activation of Cdk5/p35 attenuates PXR-mediated gene expression}

Flavonoids have been shown to inhibit protein kinases, including Cdks $[19,20]$. Flavonoids may regulate PXR by inhibiting $\mathrm{Cdk} 2$, as $\mathrm{Cdk} 2$ has been shown to negatively regulate PXR [9]. However, because flavonoids can inhibit Cdk5 [20] and Cdk5/p35 signaling (which is not regulated by cell cycling) is active in hepatoma [25], we tested whether inhibition of $\mathrm{Cdk} 5$ by flavonoids is responsible for the flavonoids-mediated activation of PXR.

Since the activity of Cdk5 requires p35 as a critical regulatory subunit, we determined whether p35 is expressed in HepG2 cells, in which flavonoid-mediated activation of PXR was first discovered. We found that p35 was expressed in HepG2 cells at levels comparable to those in 
A

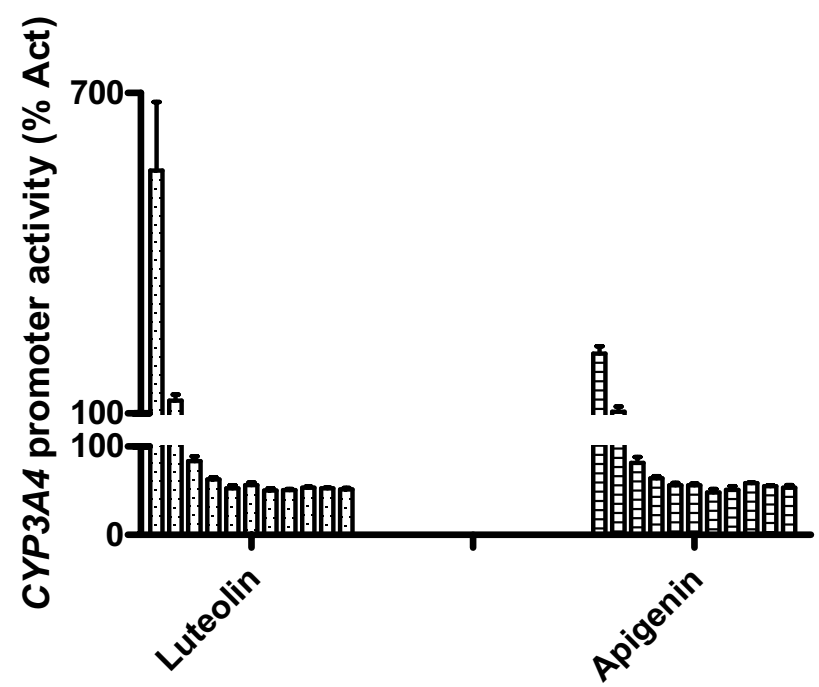

B

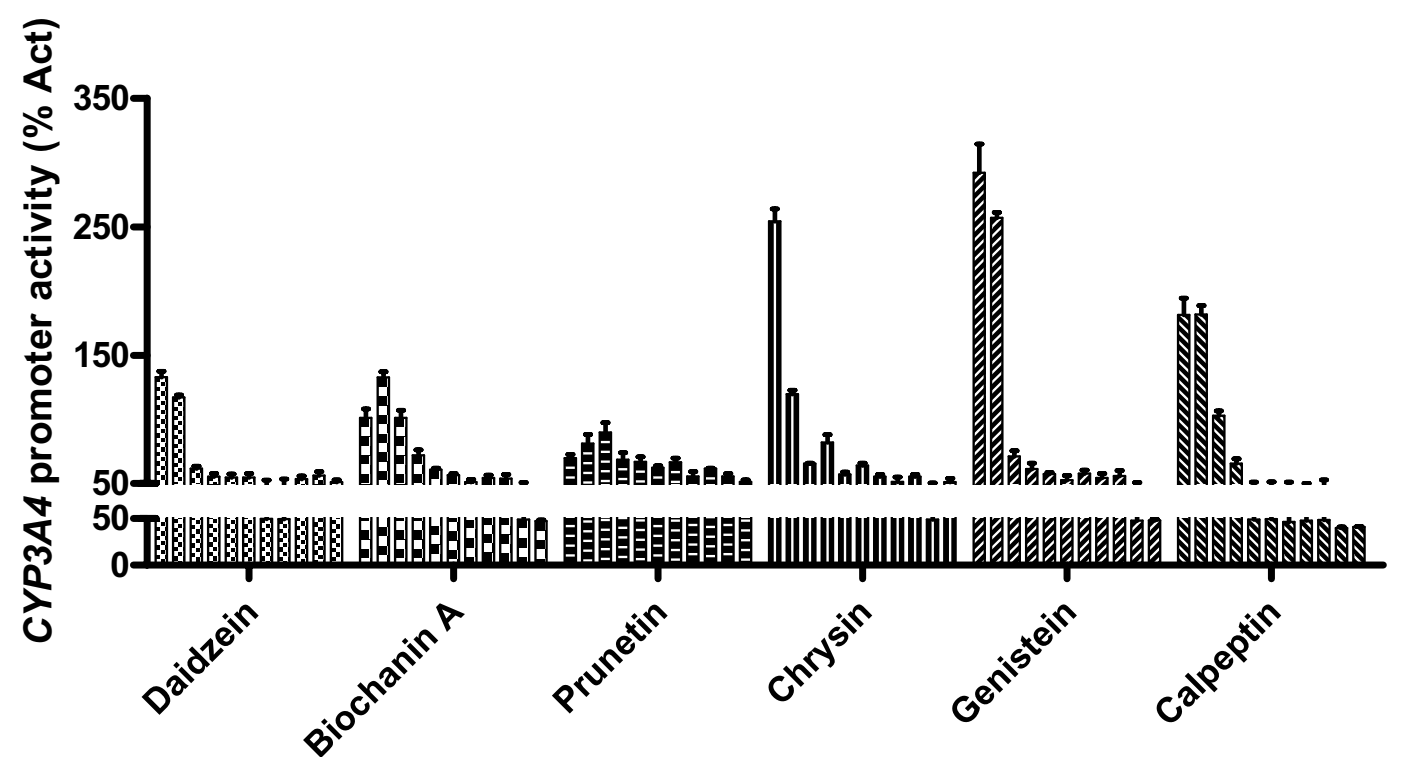

Figure 1 Flavonoids activate PXR-mediated CYP3A4 gene expression. HepG2 cells $\left(2 \times 10^{4} /\right.$ well) stably transfected with PXR and CYP3A4-luC [9] were seeded in 384-well plates and treated with indicated compounds for $24 \mathrm{~h}$ before the luciferase assay. CYP3A4 promoter activity induced by compound was expressed as a percentage of activation (\% Act) by normalizing with luciferase activity from $2 \mu \mathrm{M}$ rifampicin. Compounds were tested at 11 concentrations starting at $40 \mu \mathrm{M}$ (leftmost for each compound), 1:1.5 (A) or 1:3 (B) serial dilutions. In (A), the concentrations for each compound tested were $40,26.667,17.778,11.852,7.901,5.267,3.512,2.341,1.561,1.040$ and $0.694 \mu \mathrm{M}$ (from left to right). In (B), the concentrations for each compound tested were $40,13.333,4.444,1.481,0.494,0.165,0.055,0.018,0.006,0.002$ and $0.0007 \mu \mathrm{M}$ (from left to right). Compounds were tested in quadruplicate. The bars denote the standard deviation.

IMR-32 (Fig. 2B), a neuronal cell line that expresses p35 and has been used as a positive control for p35 expression [25].

Next, we determined the functional correlation between the activities of Cdk5 and PXR. Overexpression of Cdk5 led to attenuation of both basal (DMSO control) and rifampicin-induced activation of PXR (Fig. 3A).
Expression levels of PXR were not affected by overexpression of Cdk5 (Fig. 3B), confirming that the attenuation of PXR activity is because of the inhibitory effect of Cdk5 on PXR and not because of a decrease in expression level of PXR. The inhibitory effect of Cdk5 on PXR was further confirmed by the increase in PXR activity on siRNAmediated downregulation of Cdk5 (Fig. 3C). Reduced 


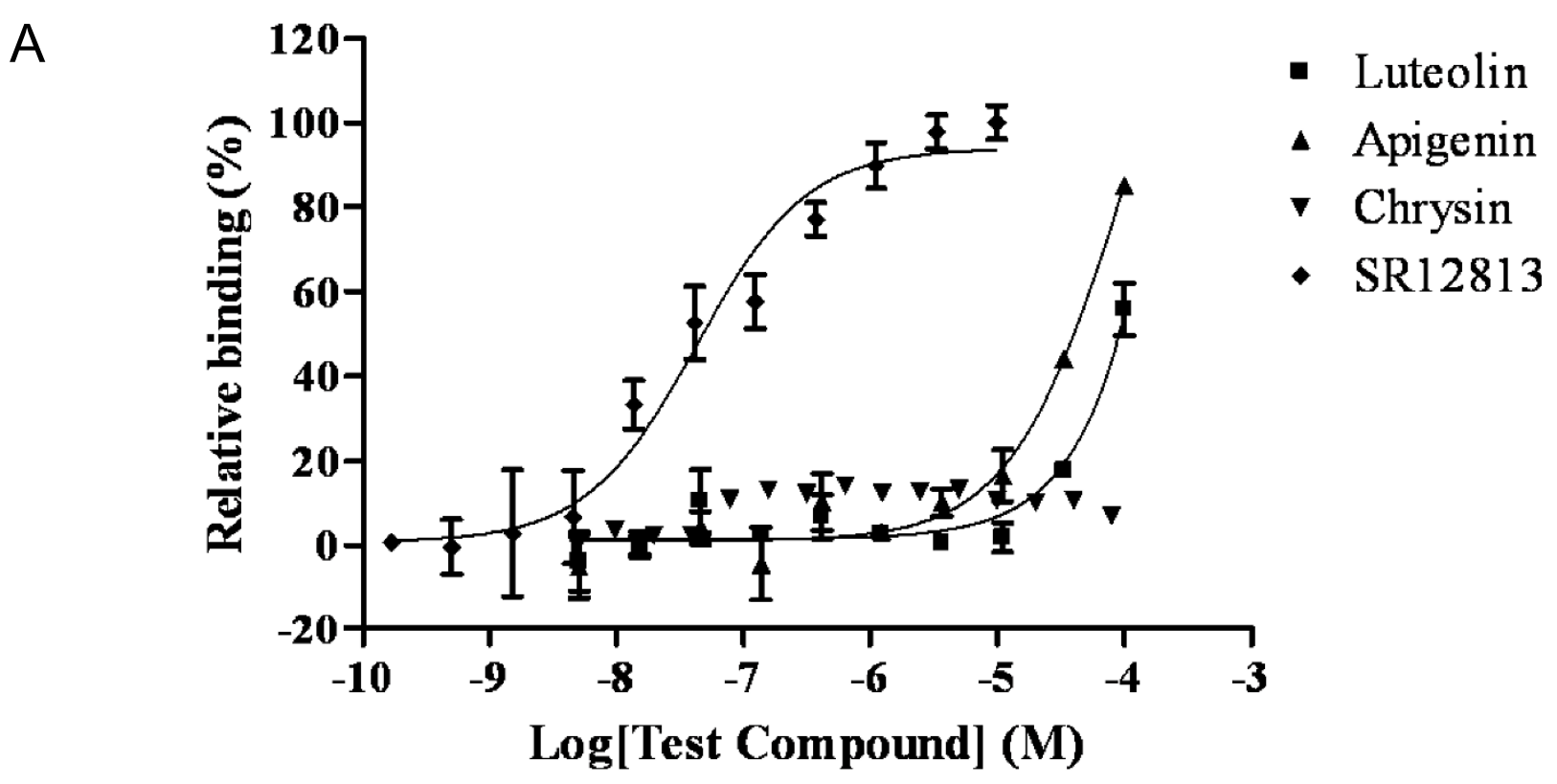

B

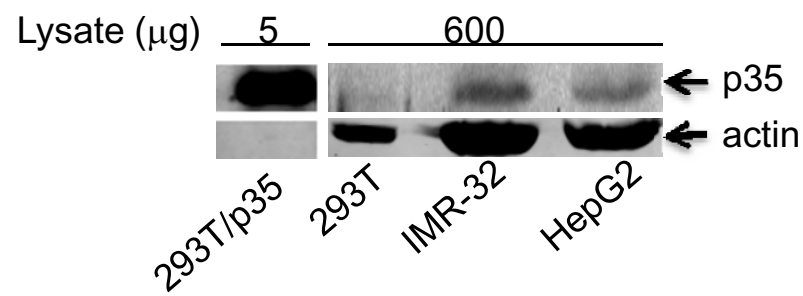

Figure 2 Chrysin, apigenin, and luteolin are not potent PXR binders; p35 is expressed in HepG2. (A) Chrysin, apigenin, and luteolin are not potent PXR binders. SR12813, a potent human PXR agonist, was used as a positive control. Relative binding (\%) was determined as described in Methods. Compounds were tested in triplicate. The bars denote the standard deviation. (B) p35 is expressed in HepG2 (lane 4). HEK 293T (293T) cells transfected with a p35 expression construct (293T/p35) $\left(8 \times 10^{5}\right.$ cells in 6-well plates transfected with 1 Mg of pCMV-mycP35 using Fugene 6) was used as a positive control for p35 expression (lane 1). 293T was used as a negative control (lane 2). IMR-32, a neuroblastoma cell line that expresses p35 endogenously, was used as an additional positive control (lane 3$)$. Total amount of lysate $(\mu \mathrm{g})$ used is indicated above the samples. Since only $5 \mu \mathrm{g}$ of total protein was used in lane 1, no actin band was detected.

expression of Cdk5 in response to siRNA treatment was verified (Fig. 3D). In addition, we showed that flavonoids significantly decreased the inhibitory effect of Cdk5 on CYP3A4 promoter activity induced by rifampicin (Fig. 4). In the absence of flavonoids, Cdk5 inhibited CYP3A4 promoter activity by $40 \%$. The inhibitory effect of Cdk 5 was decreased to $4 \%$ and $23 \%$ by $20 \mu \mathrm{M}$ of biochanin $\mathrm{A}$ and $20 \mu \mathrm{M}$ of chrysin, respectively (Fig. 4B). These results suggest that flavonoids may inhibit Cdk5 and restore the Cdk5-mediated downregulation of $C Y P 3 A 4$ promoter activity.

To further validate the role of Cdk5 in regulating PXR function, we examined the effect of calpeptin on PXR function. Calpeptin has been shown to block the conversion of p35 to the highly active p25, thereby reducing the activity of Cdk5 [23,24]. Therefore we anticipated that the calpeptin-mediated inhibition of Cdk5 would lead to activation of PXR, and calpeptin may restore the Cdk5-mediated downregulation of CYP3A4 promoter activity. Indeed, we found that calpeptin induced PXR activity (e.g., $4.4 \mu \mathrm{M}$ of calpeptin is as potent as $2 \mu \mathrm{M}$ of rifampicin) (Fig. 1B), and significantly $(p<0.001)$ decreased the inhibitory effect of $\mathrm{Cdk} 5$ on the activity of $C Y P 3 A 4$ promoter (Fig. 4B). Taken together, these data indicate that Cdk5 negatively regulates PXR activity, and that inhibi- 


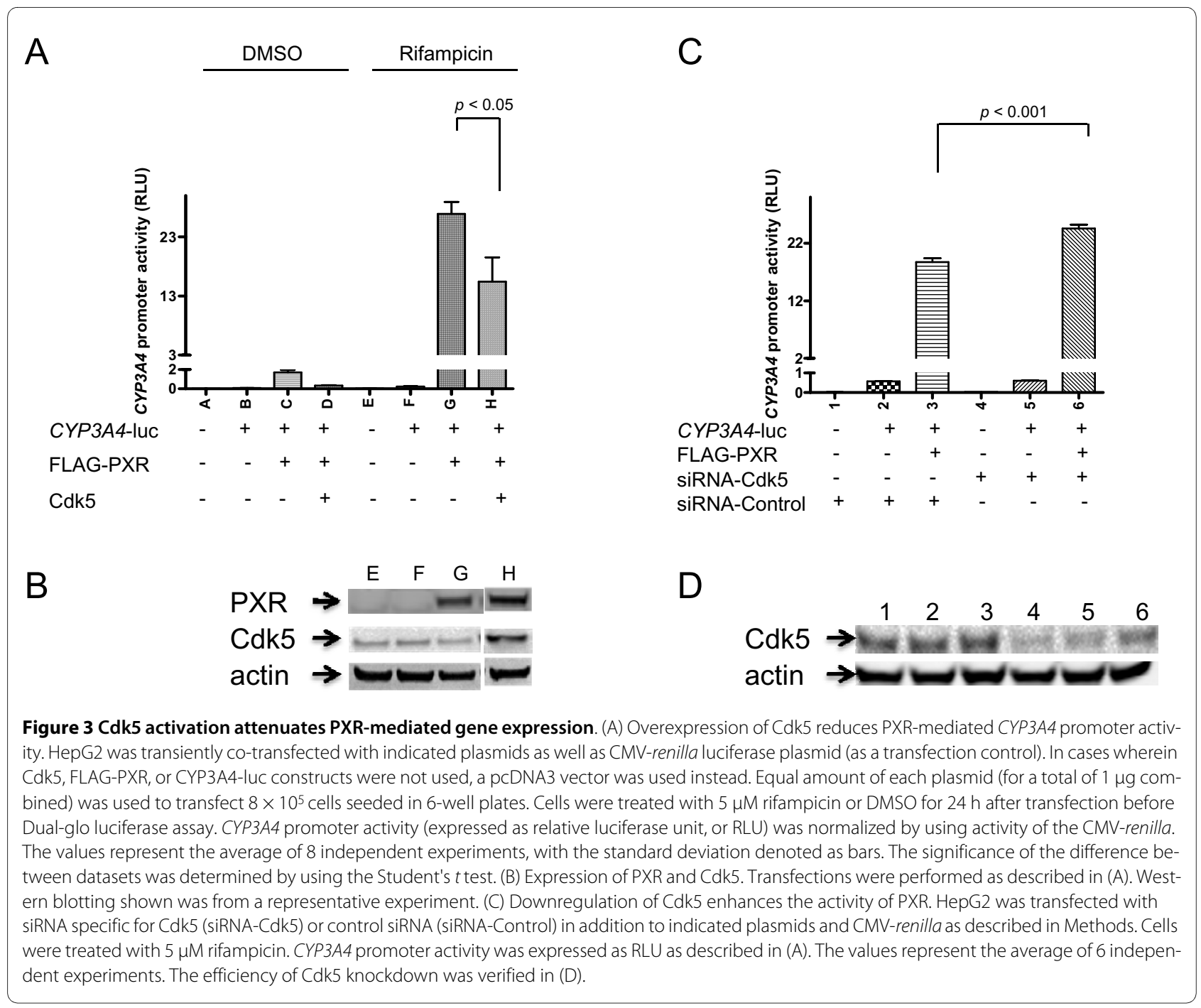

tion of Cdk5 is at least partially responsible for flavonoids-induced activation of PXR.

\section{Cdk5 phosphorylates PXR}

One possible mechanism by which Cdk5 regulates PXR is by directly phosphorylating PXR. All Cdks recognize the same motif for phosphorylation, and Cdk2 [9] and Cdk1 [37] have been shown to phosphorylate PXR. As expected, in an in vitro kinase assay, reconstituted complexes of purified Cdk $5 / \mathrm{p} 35$ directly phosphorylated PXR (Fig. 5), suggesting that Cdk5 can directly phosphorylate hPXR.

Inhibition of multiple Cdks might contribute to flavonoidsmediated activation of PXR

Since flavonoids have been reported to inhibit multiple Cdks, we investigated the inhibitory effect of flavonoid apigenin on various Cdks. Apigenin inhibited multiple Cdks, including Cdk2, 4, 5, 7, 8, 9 and 11 (Table 1). Since Cdk2 has been previously shown to negatively regulate
PXR function [9], these data suggest that inhibition of multiple Cdks might contribute to the activating effect of flavonoids on PXR.

\section{Discussion}

The widespread use of flavonoids has triggered several studies to investigate the molecular mechanisms of action of these naturally occurring compounds. Flavonoids have been reported to inhibit protein kinases such as Cdks [20] and induce the expression of drug-metabolizing enzymes such as CYPs [31-36]. The stimulatory effect of flavonoids on CYP expression might have significant implication on the pharmacokinetics of drugs co-administered with herbal remedy and potential herbal-drug interactions.

In a cell-based screening approach designed to identify activators of PXR, we identified that flavones luteolin, apigenin and chrysin and isoflavones daidzein, biochanin A, prunetin, and genistein are activators of PXR-medi- 
A

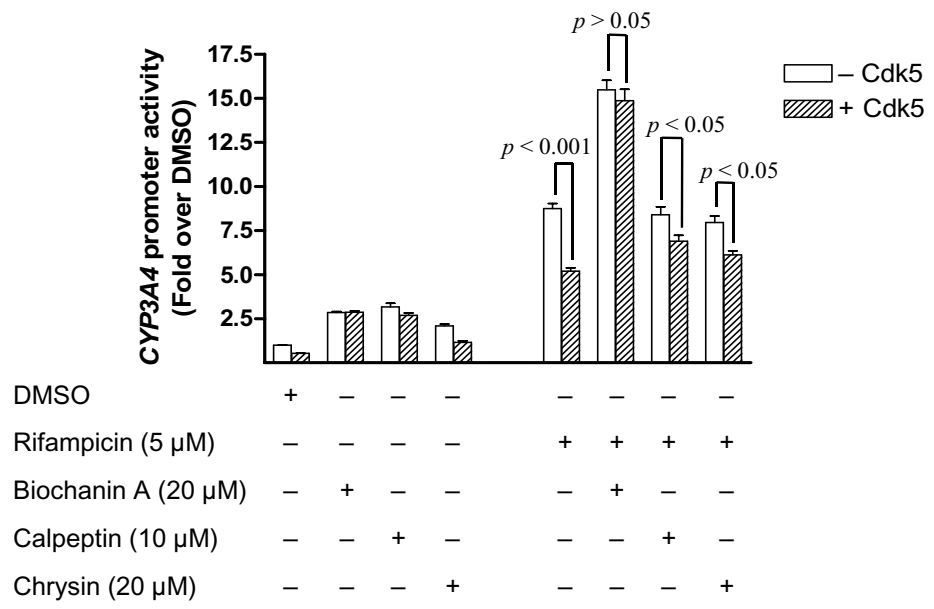

B

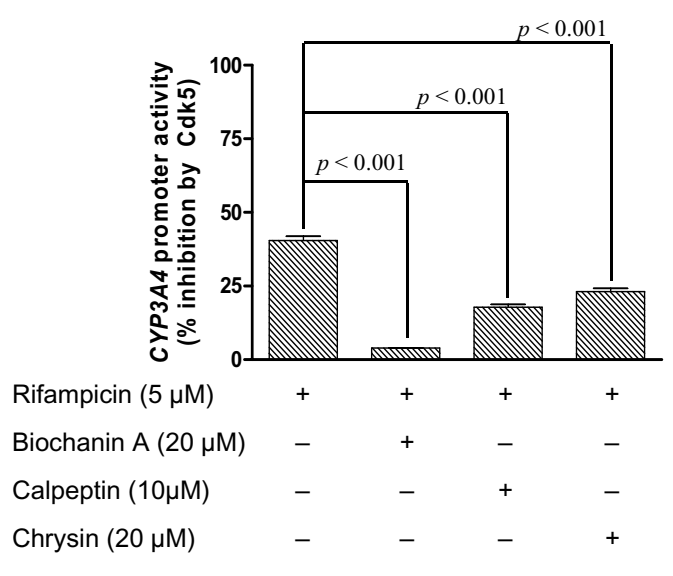

Figure 4 Flavonoids and calpeptin decrease the inhibitory effect of Cdk5 on PXR-mediated CYP3A4 promoter activity. (A) HepG2 was transiently co-transfected with FLAG-PXR, CYP3A4-luc, CMV-renilla luciferase (as a transfection control), and either Cdk5 (+ Cdk5) or pcDNA3 vector (-Cdk5) plasmids at a ratio of 1:15:1:3, respectively. A total of $20 \mu \mathrm{g}$ of combined plasmids was used to transfect $5 \times 10^{6}$ cells seeded in a T75 flask $16 \mathrm{~h}$ before transfection. Transfected cells were seeded into white 96-well plates (at 10,000 cells/well) $24 \mathrm{~h}$ later, and treated with DMSO or compounds as indicated for $24 \mathrm{~h}$ before Dual-glo luciferase assay. CYP3A4 promoter activity was normalized by using activity of the CMV-renilla, and expressed as fold induction over control (DMSO; without Cdk5 transfection). The values represent the average of 8 independent experiments, with the standard deviation denoted as bars. The significance of the difference between datasets was determined by using the Student's $t$ test. (B) Using fold induction over control as described in (A), the C dk5-mediated inhibition of CYP3A4 promoter activity was expressed a percentage of inhibition by Cdk5 [\% inhibition $=100 \% \times($ signal without C dk5 - signal with Cdk5)/signal without Cdk5)].

ated CYP3A4 gene expression. Genistein and daidzein have been previously reported to activate PXR [35]. In our study, the lack of potent binding of chrysin, luteolin and apigenin to PXR suggests that mechanisms other than direct PXR binding might be responsible for PXR activation by these flavonoids, and the reported inhibitory effect of flavonoids on Cdks (such as Cdk5) led us to investigate the functional relationship between inhibition of Cdk5 and activation of PXR.

We first showed that $\mathrm{p} 35$, a critical regulatory protein for Cdk5, is expressed in the human liver carcinoma cell line HepG2. We found an inverse correlation between Cdk5 activity and PXR activity: downregulation of Cdk/

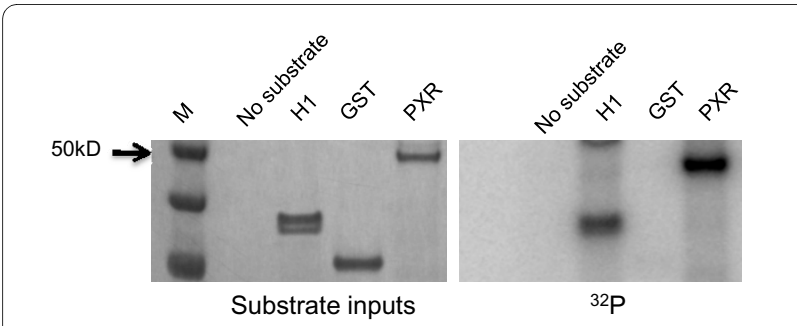

Figure 5 Cdk5 phosphorylates PXR in vitro. Kinase assays were performed as described under Methods. Histone $\mathrm{H} 1(\mathrm{H} 1)$ was the positive and GST the negative control. The amount of substrates loaded to PAGE (substrate inputs) was illustrated by SimplyBlue staining. M, molecular weight marker. p35 signaling activated whereas its upregulation inhibited PXR. In addition, flavonoids restored the Cdk5-mediated downregulation of $C Y P 3 A 4$ promoter activity. We further showed that Cdk5/p35 directly phosphorylated PXR.

$\mathrm{Cdk} 5$, unlike its regulatory subunit $\mathrm{p} 35$, is ubiquitously expressed. The expression of p35 is highest in the nervous system, and has been reported in many non-CNS

Table 1: Apigenin inhibits multiple Cdks.

\begin{tabular}{cc}
\hline Kinase & Kd $(\boldsymbol{\mu M})$ \\
\hline Cdk2 & 9.8 \\
\hline Cdk3 & $>30$ \\
\hline Cdk4 & 7.0 \\
\hline Cdk5 & 3.9 \\
\hline Cdk7 & 1.7 \\
\hline Cdk8 & 5.8 \\
\hline Cdk9 & 2.0 \\
\hline
\end{tabular}


cells and tissues such as lens epithelia [27], muscle tissues [28] hepatoma cells [25], adipose tissues [29] and male reproductive system [30]. Our discovery that p35 is expressed in HepG2 human liver carcinoma cells expands the list of cells and tissues that are found to express p35. p35 can be cleaved to generate the highly active p 25 and we show that calpeptin, a peptide previously reported to inhibit the cleavage of p35 [23,24], highly induced PXR activity and blocked the inhibitory effect of Cdk5 on PXR, supporting that Cdk5 negatively regulates PXR activity.

As with other Cdk inhibitors, the inhibitory effect of flavonoids is not specific to Cdk5, as suggested by inhibition of multiple Cdks by apigenin in the Cdk kinase profiling assay. Cdk2 has been previously shown to negatively regulate PXR function [9]. Our data suggest that flavonoid-mediated activation of PXR is not because of the inhibition of Cdk5 only; inhibition of multiple Cdks, including Cdk2, might contribute to this activation.

Gene expression of CYP3A4 is regulated not only by PXR but also by other signaling pathways including other nuclear receptors. These signaling pathways might also cross-talk with each other. Therefore, it is important to investigate the regulation of other signaling pathways and nuclear receptors by flavonoids and the implications in the regulation of gene expression of CYP $3 A 4$ and other CYPs. It is also possible that metabolites of flavonoids may play roles in this complex regulation network. Comprehensively investigating the signaling network regulated by flavonoids and their metabolites will contribute to understanding the roles of flavonoids in potential herbal-drug interactions.

\section{Conclusions}

In conclusion, this is the first report that correlates the effect of flavonoids on regulation of expression of drugmetabolizing enzymes to their inhibitory effect on Cdks, which in turn negatively regulates PXR function. Because of the widespread use of flavonoids by humans as dietary constituents, our discovery may have important implications on the pharmacokinetics of drugs co-administered with herbal remedy and herbal-drug interactions.

\section{Methods}

\section{Compounds, antibodies, and other materials}

Cell culture reagents were obtained from Invitrogen (Carlsbad, CA); compounds and anti- $\beta$-actin antibody from Sigma-Aldrich (St. Louis, MO); purified Cdk5-p35 complex and ATP from Millipore (Billerica, MA); purified human PXR protein from Origene Tech (Rockville, MD); [ $\left.\gamma^{-32} \mathrm{P}\right]$ ATP from PerkinElmer Life Sciences (Waltham, MA); charcoal/dextran-treated FBS from Hyclone (Logan, UT); Bradford reagent from Bio-Rad (Hercules, CA); and anti-Cdk5 (sc-249) and anti-p35 (sc-
821) antibodies from Santa Cruz Biotechnology (Santa Cruz, CA).

\section{Cell lines, plasmids and transfection}

HepG2 liver carcinoma cells, IMR-32 neuroblastoma cells, and HEK 293T kidney epithelial cells were obtained from the American Type Culture Collection (ATCC, Manassas, VA). All cells were maintained at $37^{\circ} \mathrm{C}$ in a humidified atmosphere containing $5 \% \mathrm{CO}_{2}$. HepG2 and HEK293T were maintained in modified Eagle's minimal essential medium (MEM) from ATCC with 10\% FBS, 2 $\mathrm{mM}$ L-glutamine and $100 \mathrm{U} / \mathrm{ml}$ penicillin/streptomycin. IMR-32 was maintained in modified Eagle's MEM supplemented with $10 \%$ FBS. The Cdk5 expression construct (Cdk5-HA) was provided by Dr. Sander van den Heuvel (Addgene plasmid 1872; Addgene, Cambridge, MA). pCMV-mycP35 was provided by Dr. Li-huei Tsai (Addgene plasmid 1347). FLAG-PXR and CYP3A4-luc plasmids were constructed as previously described [9]. Transfections were conducted with FuGENE 6 (Roche Diagnostics), according to manufacturer's instructions.

\section{PXR transactivation assay}

Compounds were added to 384-well plates seeded with cells in a phenol red-free medium containing $5 \%$ charcoal/dextran-treated FBS and incubated for $24 \mathrm{~h}$ at $37^{\circ} \mathrm{C}$ before conducting the luciferase assay. The final concentration of DMSO in each well was maintained at $0.1 \%$. DMSO and $2 \mu \mathrm{M}$ of rifampicin were used as the negative and positive control, respectively. Luciferase activities were detected by using EnVision plate reader (PerkinElmer Life Sciences), as previously reported [9].

\section{PXR binding assay}

The time-resolved fluorescence resonance transfer (TRFRET) PXR competitive binding assay was performed as described previously [9]. Briefly, the assays were performed in a volume of $20 \mu \mathrm{l}$ in 384-well solid black plates with $5 \mathrm{nM}$ GST-hPXR ligand-binding domain, $40 \mathrm{nM}$ fluorescent-labeled hPXR agonist (Fluomore PXR Green), 5 nM terbium-labeled anti-GST antibody, and test compound at different concentrations. The reaction mixture was incubated at $25^{\circ} \mathrm{C}$ for $30 \mathrm{~min}$ and then fluorescent emissions of each well were measured at $495 \mathrm{~nm}$ and 520 $\mathrm{nm}$, using an excitation filter of $340 \mathrm{~nm}$, a delay time of $100 \mu \mathrm{s}$, and an integration time of $200 \mu \mathrm{s}$ on a PHERAStar plate reader (BMG Labtech, Durham, NC). The FRET ratio was calculated by dividing the emission signal at 520 $\mathrm{nm}$ by that at $495 \mathrm{~nm}$. DMSO was used as the negative control ( $0 \%$ relative binding) and $10 \mu \mathrm{M}$ SR-12813, a human PXR agonist, as the positive control (100\% relative binding). The data were expressed as relative binding (\%) [relative binding $(\%)=100 \% \times($ DMSO FRET ratio - Compound FRET ratio)/(DMSO FRET ratio - $10 \mu \mathrm{M} \mathrm{SR12813}$ 
FRET ratio)]. Curves were generated by using GraphPad Prism 4.0 (GraphPad Software, La Jolla, CA).

\section{siRNA knockdown}

Endogenous Cdk5 was knocked down by using ON-TARGETplus SMARTpool Cdk5 siRNA (L-003239-00; Thermo Scientific, Chicago, IL). Knockdown efficiency of the target gene was confirmed by Western blotting, as described previously [9]. Briefly, HepG2 cells $\left(2 \times 10^{5}\right)$ were seeded in 6-well plates in serum-free Eagle's MEM. Cells were transfected with 100 pmol siRNA, using Lipofectamine 2000 (Invitrogen). After $6 \mathrm{~h}$, the medium was replaced by Eagle's MEM containing 10\% FBS and cells were allowed to grow uninterrupted for $42 \mathrm{~h}$. Cells were then transfected with a total of $1 \mu \mathrm{g}$ of plasmid DNA by using Fugene 6, cultured for another $24 \mathrm{~h}$, and treated and processed for the luciferase assay or Western blotting.

\section{In vitro Cdk kinase assay}

For the in vitro kinase assay, 20 ng of recombinant Cdk5/ p35 was used per reaction. Kinase assays were performed in $25 \mu \mathrm{l}$ reactions, with $1 \mu \mathrm{g}$ substrate protein PXR, 0.5 $\mu \mathrm{mol} / \mathrm{l}$ cold ATP and $5 \mu \mathrm{Ci}\left[\gamma^{-32} \mathrm{P}\right]$ ATP $(6000 \mathrm{Ci} / \mathrm{mmol})$. GST was expressed and purified by using pGEX-4T-1 in Escherichia coli BL21 and was used as the negative control. The reaction mixture was incubated at $30^{\circ} \mathrm{C}$ for 30 min before being electrophoresed by SDS-PAGE. The gel was stained by using SimplyBlue SafeStain (Invitrogen), desiccated by the Labconco gel dryer (Labconco, Kansas City, MO), and exposed overnight to the Storage Phosphor Screen (GE Healthcare). Phosphoimages were obtained by using the Storm scanner (GE Healthcare). In vitro Cdks kinase profiling assays were performed by Ambit Biosciences (San Diego, CA) as previously described [38,39].

\section{Statistical analyses}

Results are expressed as the mean $\pm \mathrm{SD}$ of at least three independent experiments as indicated. The Student's $t$ test for the paired samples was used to determine statistical significance of difference between parameters. Differences were considered significant for $p<0.05,0.01$ or 0.001 and non-significant for $p>0.05$.

\section{Abbreviations}

PXR: pregnane $\times$ receptor; CYP: cytochrome P450; Cdk: cyclin-dependent kinase.

\section{Authors' contributions}

HD carried out the transactivation, Western blotting and kinase assays and corresponding data analysis and drafted the manuscript. WL carried out the compound screening and binding assays and corresponding data analysis. JW carried out the construction of plasmids and stable cell line. JW and WL also analyzed the effect of flavonoids and calpeptin on restoring the Cdk5-mediated attenuation of PXR function. TC conceived of and coordinated the design and implementation of the study, and wrote the final manuscript. All authors read and approved the final manuscript.

\section{Acknowledgements}

We thank Drs. van den Heuvel and Li-huei Tsai for plasmids; members of the Chen group for reagents and their valuable discussions; and Dr. Kip Guy for reviewing and Dr. Vani Shanker for editing the manuscript.

This work was supported by the American Lebanese Syrian Associated Charities, St. Jude Children's Research Hospital, National Institute of General Medical Sciences grant RO1GM086415 (to T.C.), and National Cancer Institute grant P30CA027165.

\section{Author Details}

'Department of Chemical Biology and Therapeutics, St. Jude Children's Research Hospital, 262 Danny Thomas Place, Memphis, TN 38105, USA and 2Albert Einstein College of Medicine, 1300 Morris Park Ave., Ullmann 609, Bronx, NY 10461, USA

Received: 8 January 2010 Accepted: 16 June 2010

Published: 16 June 2010

\section{References}

1. Kliewer SA, Moore JT, Wade L, et al:: An orphan nuclear receptor activated by pregnanes defines a novel steroid signaling pathway. Cell 1998, 92:73-82.

2. Gong H, Sinz MW, Feng Y, et al:: Animal models of xenobiotic receptors in drug metabolism and diseases. Methods Enzymol 2005, 400:598-618.

3. Zhou SF: Drugs behave as substrates, inhibitors and inducers of human cytochrome P450 3A4. Curr Drug Metab 2008, 9:310-322.

4. Timsit YE, Negishi M: CAR and PXR: the xenobiotic-sensing receptors. Steroids 2007, 72:231-246.

5. Pondugula $\mathrm{S}$, Dong $\mathrm{H}, \mathrm{Chen} \mathrm{T}$ : Phosphorylation and protein-protein interactions in PXR-mediated CYP3A repression. Expert Opin Drug Metab Toxicol 2009, 5:861-873.

6. Ding X, Staudinger JL: Repression of PXR-mediated induction of hepatic CYP3A gene expression by protein kinase C. Biochem Pharmacol 2005, 69:867-873.

7. Ding $X$, Staudinger JL: Induction of drug metabolism by forskolin: the role of the pregnane $\times$ receptor and the protein kinase a signal transduction pathway. J Pharmacol Exp Ther 2005, 312:849-856.

8. Lichti-Kaiser K, Xu C, Staudinger JL: Cyclic AMP-dependent protein kinase signaling modulates pregnane $\times$ receptor activity in a speciesspecific manner. J Biol Chem 2009, 284:6639-6649.

9. Lin W, Wu J, Dong H, et al.: Cyclin-dependent kinase 2 negatively regulates human pregnane $\times$ receptor-mediated CYP3A4 gene expression in HepG2 liver carcinoma cells. J Biol Chem 2008, 283:30650-30657

10. Pondugula S, Brimer-Cline C, Wu J, et al: : p phosphomimetic mutation at threonine-57 abolishes transactivation activity and alters nuclear localization pattern of human pregnane $\times$ receptor. Drug Metab Dispos 2009, 37:719-730.

11. Kodama S, Koike C, Negishi M, et al:: Nuclear receptors CAR and PXR cross talk with FOXO1 to regulate genes that encode drugmetabolizing and gluconeogenic enzymes. Mol Cell Biol 2004 24:7931-7940

12. Zhou C, Tabb MM, Nelson EL, et al: Mutual repression between steroid and xenobiotic receptor and NF-kappaB signaling pathways links xenobiotic metabolism and inflammation. J Clin Invest 2006, 116:2280-2289.

13. GuX, Ke S, Liu D, et al:: Role of NF-kappaB in regulation of PXR-mediated gene expression: a mechanism for the suppression of cytochrome $\mathrm{P}$ 4503 A4 by proinflammatory agents. J Biol Chem 2006, 281:17882-17889.

14. Xie W, Tian Y: Xenobiotic receptor meets NF-kappaB, a collision in the small bowel. Cell Metab 2006, 4:177-178.

15. Spencer JP: Flavonoids: modulators of brain function? Br J Nutr 2008 99:ES60-77.

16. Ververidis F, Trantas E, Douglas C, et al:: Biotechnology of flavonoids and other phenylpropanoid-derived natural products. Part II: Reconstruction of multienzyme pathways in plants and microbes. Biotechnol J 2007, 2:1235-1249.

17. Yamamoto Y, Gaynor RB: Therapeutic potential of inhibition of the NF$\mathrm{KB}$ pathway in the treatment of inflammation and cancer. J Clin Inves 2001, 107:135-142. 
18. Cushnie TP, Lamb AJ: Antimicrobial activity of flavonoids. Int $\mathrm{J}$ Antimicrob Agents 2006, 26:343-356.

19. Casagrande F, Darbon JM: Effects of structurally related flavonoids on cell cycle progression of human melanoma cells: regulation of cyclindependent kinases CDK2 and CDK1. Biochem Pharmacol 2001, 61:1205-1215.

20. Zapata-Torres G, Opazo F, Salgado C, et al:: Effects of natural flavones and flavonols on the kinase activity of Cdk5. J Nat Prod 2004, 67:416-420.

21. Dhavan R, Tsai LH: A decade of Cdk5. Nat Rev Mol Cell Biol 2001, 2:749-759.

22. Patrick GN, Zukerberg L, Nikolic M, et al.: Conversion of p35 to p25 deregulates $\mathrm{Cdk} 5$ activity and promotes neurodegeneration. Nature 1999, 402:615-622.

23. Lee MS, Kwon YT, Li M, et al: Neurotoxicity induces cleavage of $\mathrm{p} 35$ to p25 by calpain. Nature 2000, 405:360-364.

24. Lin H, Juang $\mathrm{JL}$, Wang PS: Involvement of Cdk5/p25 in digoxin-triggered prostate cancer cell apoptosis. J Biol Chem 2004, 279:29302-29307.

25. Selvendiran $\mathrm{K}$, Koga $\mathrm{H}$, Ueno $T$, et al:: Luteolin promotes degradation in signal transducer and activator of transcription 3 in human hepatoma cells: an implication for the antitumor potential of flavonoids. Cancer Res 2006, 66:4826-4834.

26. Tang D, Lee KY, Qi Z, et al:: Neuronal Cdc2-like kinase: from cell cycle to neuronal function. Biochem Cell Biol 1996, 74:419-429.

27. Gao CY, Zakeri Z, Zhu Y, et al:: Expression of Cdk5, p35 and Cdk5associated kinase activity in the developing rat lens. Dev Genet 1997, 20:267-275

28. Lazaro JB, Kitzmann M, Poul MA, et al:: Cyclin dependent kinase 5, Cdk5, is a positive regulator of myogenesis in mouse C2 cells. J Cell Sci 1997, 110:1251-1260.

29. Okada S, Yamada E, Saito T, et al:: Cdk5-dependent phosphorylation of the Rho family GTPase TC10a regulates insulin-stimulated GLUT4 translocation. J Biol Chem 2008, 283:35455-35463.

30. Lin H, Chen MC, Ku CT: Cyclin-dependent kinase 5 regulates steroidogenic acute regulatory protein and androgen production in mouse Leydig cells. Endocrinology 2009, 150:396-403.

31. Liu DY, Yang M, Zhu HJ, et al:: Human pregnane $\times$ receptor-mediated transcriptional regulation of cytochrome $\mathrm{P} 4503 \mathrm{~A} 4$ by some phytochemicals. Zhejiang Da Xue Xue Bao Yi Xue Ban 2006, 35:8-13.

32. Kluth $D$, Banning A, Paur I, et al:: Modulation of pregnane $\times$ receptor-and electrophile responsive element-mediated gene expression by dietary polyphenolic compounds. Free Radic Biol Med 2007, 42:315-325.

33. Satsu H, Hiura Y, Mochizuki K, et al:: Activation of pregnane $\times$ receptor and induction of MDR1 by dietary phytochemicals. J Agric Food Chem 2008, 56:5366-5373.

34. Li L, Stanton JD, Tolson AH, et al:: Bioactive terpenoids and flavonoids from Ginkgo biloba extract induce the expression of hepatic drugmetabolizing enzymes through pregnane $\times$ receptor, constitutive androstane receptor, and aryl hydrocarbon receptor-mediated pathways. Pharm Res 2009, 26:872-882.

35. Li Y, Ross-Viola JS, Shay NF, et al.: Human CYP3A4 and murine Cyp3A11 are regulated by equol and genistein via the pregnane $\times$ receptor in a species-specific manner. J Nutr 2009, 139:898-904.

36. Messina A, Nannelli A, Fiorio R, et al.: Expression and inducibility of CYP1A1, 1A2, $1 \mathrm{~B} 1$ by beta-naphthoflavone and CYP2B22, 3A22, 3A29, $3 \mathrm{~A} 46$ by rifampicin in the respiratory and olfactory mucosa of pig. Toxicology 2009, 260:47-52.

37. Lichti-Kaiser $K$, Brobst D, Xu C, et al: A systematic analysis of predicted phosphorylation sites within the human PXR protein. J Pharmacol Exp Ther 2009, 331:65-76.

38. Fabian MA, Biggs WH, Treiber DK, et al:: A small molecule-kinase interaction map for clinical kinase inhibitors. Nat Biotechnol 2005, 23:329-336

39. Karaman MW, Herrgard S, Treiber DK, et al:: A quantitative analysis of kinase inhibitor selectivity. Nat Biotechnol 2008, 26:127-132.

doi: $10.1186 / 1471-2091-11-23$

Cite this article as: Dong et al., Flavonoids activate pregnane $\times$ receptormediated CYP3A4 gene expression by inhibiting cyclin-dependent kinases in HepG2 liver carcinoma cells BMC Biochemistry 2010, 11:23

\section{Submit your next manuscript to BioMed Central} and take full advantage of:

- Convenient online submission

- Thorough peer review

- No space constraints or color figure charges

- Immediate publication on acceptance

- Inclusion in PubMed, CAS, Scopus and Google Scholar

- Research which is freely available for redistribution 